\title{
New insights into growth hormone action
}

\author{
M J Waters, H N Hoang, D P Fairlie, R A Pelekanos and R J Brown \\ Institute for Molecular Bioscience and School of Biomedical Sciences, University of Queensland, St Lucia, Australia 4072
}

(Requests for offprints should be addressed to M J Waters; Email: m.waters@imb.uq.edu.au)

\begin{abstract}
It has been 75 years since Evans and Long identified a somatic growth-promoting substance in pituitary extracts, yet it is only in the last 20 years that the molecular basis for this action has been established. Three key elements in this elucidation were the cloning of the GH receptor, the identification of Janus kinase (JAK) 2 as the receptor-associated tyrosine kinase, and the delineation of signal transduction and activators of transcription (STAT) $5 \mathrm{a} / \mathrm{b}$ as the key transcription factor(s) activated by JAK2. The interaction between these three elements results in enhanced postnatal growth and is the subject of this review. We describe a new model for GH receptor activation based on subunit rotation within a constitutive dimer, together with the phenotype and hepatic transcript profile of mice with targeted knockins to the receptor cytoplasmic domain. These support a central role for STAT5a/b in postnatal growth.
\end{abstract}

Journal of Molecular Endocrinology (2006) 36, 1-7

\section{Growth hormone (GH) receptor (GHR) activation}

While the GHR was the first of the class 1 cytokine receptors to be cloned, little useful information about its signaling mechanism was then evident other than that it being a single-pass transmembrane protein without intrinsic tyrosine kinase activity. The subsequent crystallization of the extracellular domain (ECD) of the human GHR (hGHR) complexed with hGH revealed the ability of GH to dimerize the ECD through the use of different surfaces on the hormone which bound to similar receptor domains (deVos et al. 1992). The 1:2 stoichiometry seen in the crystal structure was verified with solution studies, and when Fuh et al. (1992) subsequently reported that three monoclonal antibodies (mAbs) to the GHR ECD were able to act as receptor agonists provided they were divalent, it was natural to propose receptor activation by hormone-dependent dimerization. The finding that disruption of binding to the second receptor with a bulky substitution at Gly-120 resulted in a GH antagonist supported this view. Further, modelling studies based on hormone-dependent receptor dimerization predicted a bell-shaped doseresponse relationship, which was observed. These observations (Fuh et al. 1992) supported the liganddependent receptor dimerization model for tyrosine kinase receptors such as the epidermal growth factor receptor, and was rapidly accepted as the textbook paradigm. Further support for this model came when it

Journal of Molecular Endocrinology (2006) 36, 1-7

0952-5041/06/036-001 C 2006 Society for Endocrinology Printed in Great Britain was shown that the tyrosine kinase Janus kinase (JAK) 2 bound to a conserved proline-rich sequence of the receptor cytoplasmic domain close to the cell membrane, so that hormone-dependent dimerization would bring these two kinases into proximity, initiating transphosphorylation within the kinase-activation loop, hence JAK2 activation. JAK2 phosphorylation of selected receptor cytoplasmic domain tyrosine residues sets up binding sites for Src homology 2 (SH2) domain-containing proteins such as signal transduction and activators of transcription (STAT) 5, and JAK2 also phosphorylates other regulatory proteins.

This plausible model rests upon hormone-dependent receptor dimerization, but is this actually the case? The mAb agonist studies of Fuh et al. (1992) used a chimeric receptor stably expressed in FDCP-1 cells, where the GHR ECD was fused to the ECD, transmembrane domain (TMD) and cytoplasmic domain of the granulocyte colony-stimulating factor (G-CSF) receptor. When we repeated these experiments with a panel of mAbs to the GHR ECD and cell lines expressing full-length GHR, we found only one of $14 \mathrm{mAbs}$ to have significant agonist activity even though eight were able to dimerize and activate the chimeric receptor (Rowlinson et al. 1998). We have subsequently undertaken high-resolution epitope mapping of this $\mathrm{mAb}(\mathrm{mAb} 263)$, and shown that although it does not bind within the hormone-binding surface, it binds in a way which would promote relative receptor subunit twisting. Torsional force is evidently required, since the 
monovalent antibody fragment does not act as an agonist (Wan et al. 2003). The alternate view that receptor dimerization is particularly stringent for receptor subunit alignment is not supported by other studies which use particular mAbs to the ECD to document conformational changes which occur upon hormone-dependent activation of the receptor (Jiang et al. 2004). Hormone-dependent conformational change is also supported by protease $\mathrm{K}$ digestion studies, which show differences in susceptibility to proteolysis dependent on whether the hGH agonist or the G120R antagonist is bound (van Kerkhof et al. 2002). While it could be argued that hormone-dependent dimerization could account for the latter differences, Harding et al. (1996) showed that the G120R antagonist can form a trimeric complex at the cell surface, contrary to in vitro studies with the isolated ECDs. One final piece of evidence is not consistent with the hormone-induced dimerization model: we have recently reported that the potency of porcine GH can be increased up to 5-fold by substituting hGH residues into porcine GH. Based on the diffusion-dependent dimerization model of Pearce et al. (1999), it should not be possible to increase the biopotency of GH given the kinetic parameters evident for binding of these porcine GH analogues (Wan et al. 2004).

These disagreements with the hormone-dependent dimerization paradigm encouraged us to re-evaluate the model (Brown et al. 2005). In agreement with recent studies from Strous' group (Gent et al. 2002), we found that, in co-immunoprecipitation studies, a portion of the full-length GHR exists as constitutive dimers in vivo. This was evident using co-immunoprecipitation of hemagglutinin and FLAG N-terminally tagged receptors. However, the proportion of dimer was found to increase in the presence of hormone. This could be a result of hormone-dependent dimerization of a partially monomeric population or because hormone-binding stabilizes the trimeric complex during detergent solubilization and immunoprecipitation. This is a result of the binding energy imparted from the two hormone-receptor interactions as well as the receptor-receptor interaction in the membrane-proximal ECD (Chen et al. 1997, Bernat et al. 2003). In order to resolve this issue, we have used fluorescence and bioluminescence resonance energy transfer (FRET and BRET), techniques which can be applied to full-length receptors in the cell membrane. Using transient co-expression of receptors labelled with a fluorescence donor or an acceptor, we find high ratios consistent with constitutive dimer formation (i.e. receptor fluorophores within $100 \AA$ ). Further, by serial truncating of the receptor ECD and cytoplasmic domains, we conclude that this association is mediated by the transmembrane/juxtamembrane domain. Importantly, addition of hormone even at high concentration was without effect on the BRET and
FRET ratios in plasma membrane fractions, indicating that at the cell membrane $\mathrm{GH}$ does not induce receptor dimerization, at least for transiently expressed GHR. Appropriate controls for non-specific receptor aggregation showed that homo-dimerization of the GHR was specific.

Constitutive dimerization of the receptor implies that the activation process involves a conformational change. In order to determine the nature of this, we determined the crystal structure of the unliganded hGHR ECD, and compared it with the receptor ECDs in the 2:1 complex at $2 \cdot 7 \AA$ (Brown et al. 2005). This revealed only minor conformational change on hormone binding, notably some relative rotation of the upper and lower domains, and movement in the Trp-104 residue, the key hormone-binding residue for both receptors. Importantly, the unliganded receptor ECD does not exist as a dimer in the crystal structure, contrary to the homologous erythropoietin receptor (EPOR). This does not support involvement of a scissor movement in the activation process, currently believed to be the basis for EPOR activation (Kubatsky et al. 2001).

Lack of major conformational change on hormone binding restricts signaling options to a rotation or a plunger movement, and both are possible given that receptor 1 is rotated with respect to receptor 2 in the 2:1 crystallographic complex, as well as sitting higher than receptor 2 relative to the cell surface. To test this we have induced relative subunit rotations of $100^{\circ}$ by introducing alanines into the TMD and just below. These show that four Ala insertions are able to induce constitutive activation of JAK2 and STAT5 and proliferation seen in cell populations expressing equivalent low levels of receptor. Four Ala residues would induce a $40^{\circ}$ rotation past the initial position in a helix. These results are not consistent with a plunger movement, since both receptors would be at equivalent height to the Ala insertions. However, they are consistent with activation by relative subunit rotation, a mechanism in agreement with a study showing constitutive activation of the EPOR when receptor subunits are rotated by replacement of the ECD with a coiled coil (Seubert et al. 2003).

As illustrated in Fig. 1 we propose that identical receptor subunits are held in an equivalent and inactive orientation by $\mathrm{TMD} /$ juxtamembrane interactions, as recently described for the EPOR (Seubert et al. 2003). In this conformation, locking of the 'dimerization domains' of the ECDs is prevented by steric and charge repulsions involving Tyr-200, Pro-198, His-150 and Asp-152. Hormone binding to receptor 1 initiates activation, with the limited flexibility of the individual membrane connector sequences facilitating alignment of the second receptor through thermal movement, allowing the second site on the hormone to bind its receptor. As the second receptor-binding site is closer to the membrane, 


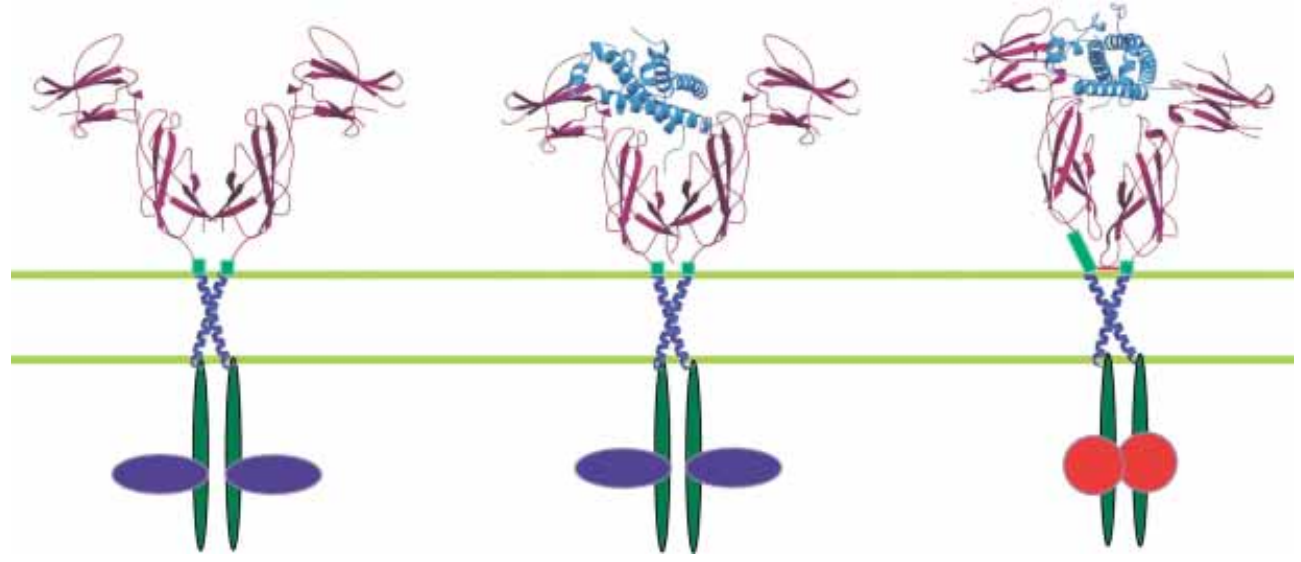

Figure 1 Model for GHR activation. GH binding to a constitutive receptor dimer results in relative rotation of receptor subunits in the homodimer, producing realignment of JAK2 kinases bound to the membrane-proximal sequence below the cell membrane. Appropriately aligned JAK2s are then able to activate each other by transphosphorylation, initiating signaling cascades. From Brown et al. (2005).

and not symmetrically placed, site 2 binding would drive the second receptor down and rotate it relative to the first receptor, allowing the 'dimerization domains' to lock together in the signaling-productive mode. The combined energy of the three binding surfaces (Bernat et al. 2003) would then be sufficient to rotate the TMD helices to the active orientation, and, given rigidity in the submembrane linker, align the box 1 sequences so that the JAK2s can transphosphorylate each other, initiating the signaling cascade. Rotation of the JAK2-binding sites (box 1) in the EPOR is sufficient for such activation (Seubert et al. 2003).

In order to provide more substantive evidence for rigidity in the submembrane linker between the TMD and the box 1 sequence, we have undertaken nuclear magnetic resonance (NMR) studies with a 24 mer peptide in water/triflouroethanol mixtures which indicate a helical propensity for this sequence (Fig. 2). This comprised four residues of the putative TMD sequence, the linker (underlined) and the box 1 sequence (Ac-VFLFSKQQRIKMLILPPVPVPKIK-NH $\mathrm{N}_{2}$ ). Total correlated spectroscopy (TOCSY) spectra at $600 \mathrm{MHz}$ were used to assign resonances and individual spin systems (not shown). Nuclear Overhauser enhancement spectroscopy (NOESY) spectra were used to identify sequential connectivity and intra-residue $\mathrm{NH}-\mathrm{CH}$ crosspeaks. The three-dimensional solution structure was calculated for the N-terminal 15 residues of the peptide using dynamic simulated annealing and energy minimization in Xplor (version 3.851) from 72 NOE (36 sequential, 30 medium-range) distance restraints, and $6 \varphi$ angle restraints $\left({ }^{3} \mathcal{F}_{\mathrm{NHCH} \alpha}<6 \mathrm{~Hz}, \varphi=65 \pm 35^{\circ}\right)$. No explicit $\mathrm{H}$-bond restraints were included in calculations. The final 15 lowest-energy structures contained no dihedral angle $\left(>5^{\circ}\right)$ or distance $(>0 \cdot 3 \AA)$ violations and are displayed in Fig 2b. These lowest energy structures a

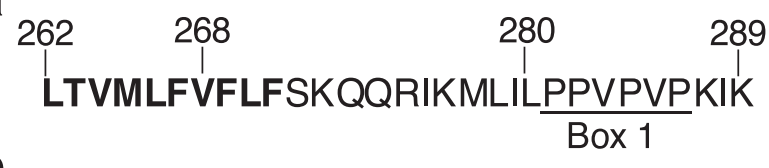

b

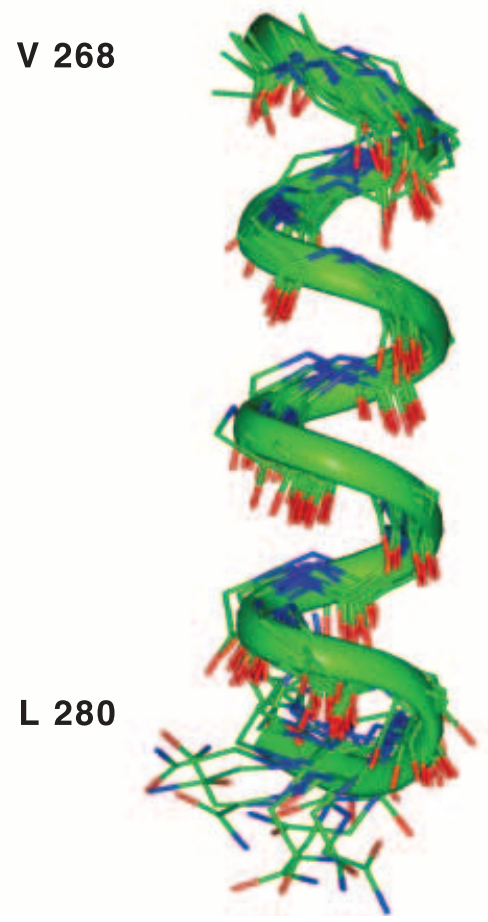

Figure 2 Secondary structure of the receptor sequence between the transmembrane domain helix and box 1 , showing $\alpha$-helical structure. (a) Predicted TMD sequence is in bold; box 1 sequence is underlined. (b) Structure obtained by NMR spectroscopy for this fragment peptide. 


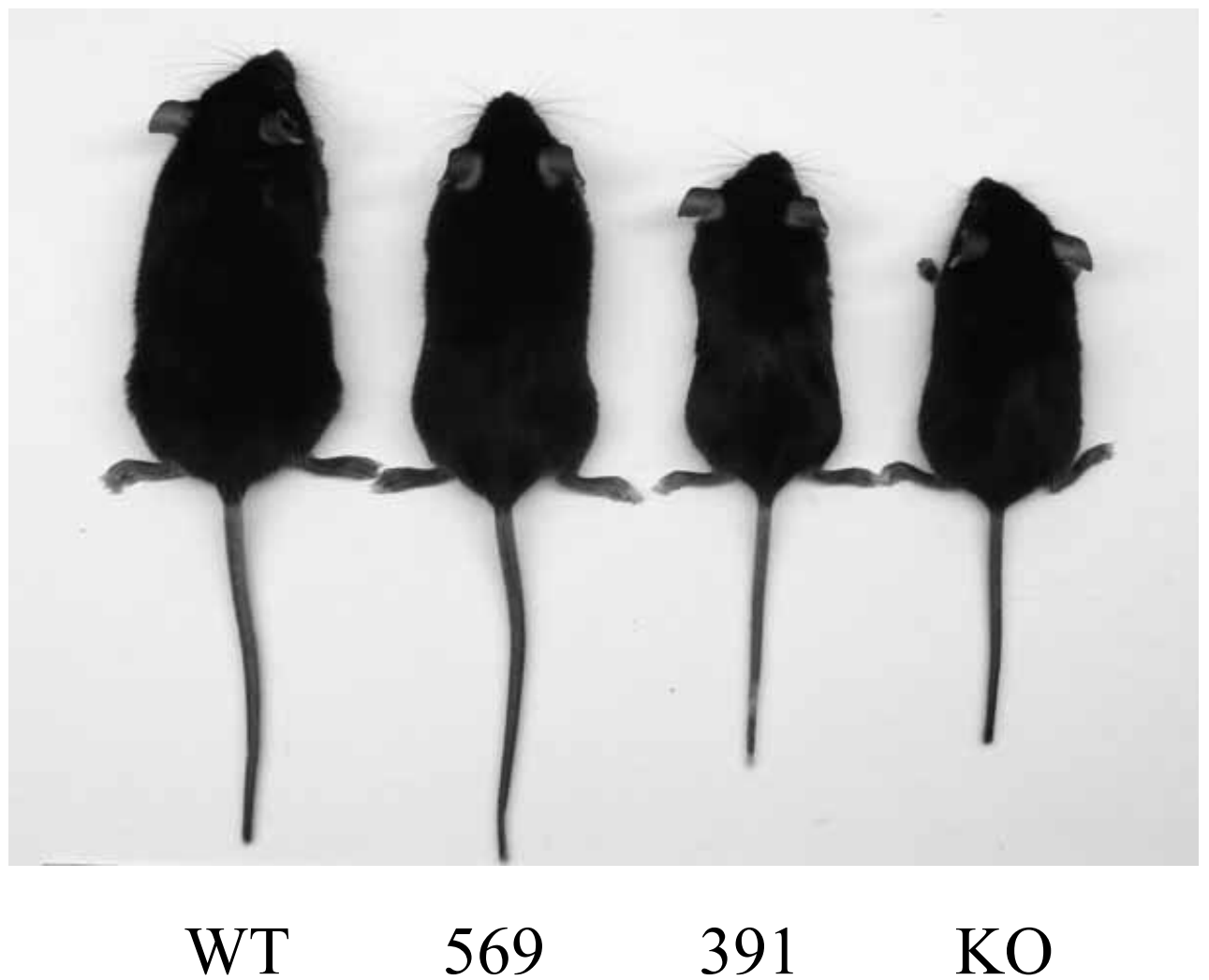

Figure 3 Growth phenotype of mice possessing homozygous mutations to the GHR cytoplasmic domain. Numbers correspond to truncation in the cytoplasmic domain. GHRKO, GHR knockout; Wt, wild type. From Rowland et al. (2005).

indicate three well-defined $\alpha$-helical turns (from $\mathrm{V} 1$ to M12), with eight $i \rightarrow i+4$ hydrogen bonds between $\mathrm{NH}$ protons of F4, K6, Q8, R9, K11, M12, L13 and I14 and CO oxygens of the N-terminal acetyl group, F2, F4, S5, Q7, Q8, R9 and I10; and a looser turn from residues (L13, I14, L15) that are neighboring the proline-rich stretch. The average backbone pairwise root mean squared deviation was $1.22 \AA$ for the helical segment. The C-terminal nine residues (PPVPVPKIK- $\mathrm{NH}_{2}$ ) displayed strong $d_{\alpha \delta}(i, i+1)$ nuclear Overhauser effects (NOEs) for L15-P16, P16-P17, V18-P19 and V20-P21 consistent with trans-amide geometry, and strong $d_{\alpha \mathrm{N}}(i+1 ; \quad i+2)$ NOEs throughout indicate $\beta$-turn conformations for the box 1 motif.

In this rotation-dependent model, the G120R hGH antagonist would be unable to correctly align the second receptor, preventing the requisite locking of the dimerization domains and rotation. This would result in increased susceptibility of the G120R hGH-bound receptor to proteolysis, as reported (van Kerkhof et al. 2002). A bell-shaped dose-response curve (Fuh et al. 1992) could result from two hormone molecules simultaneously occupying both receptor subunits, given some splaying out of the individual receptor-binding domains through vibrational movement. A bell-shaped curve has also been reported for EPOR (Schneider et al. 1997), which exists as a constitutive dimer (Kubatsky et al. 2001).

\section{The downstream growth signal}

GHR activation results in the initiation of a multiplicity of downstream signals, including STATs 1,3 and $5 \mathrm{a} / \mathrm{b}$, phosphoinositide 3-kinase/Akt, Src, extracellular-signalregulated kinase, c-Jun $\mathrm{N}$-terminal kinase and stressactivated protein kinase pathways, and increased cell calcium, demonstrable in vitro. Which of these is responsible for postnatal growth enhancement was not clear, and the absence of mutations in the cytoplasmic signaling domain of the receptor meant there were no clinical cases which could help to answer this question. The latter is surprising, considering that over 50 mutations have been identified in the receptor ECD (exons 1-7) which result in dwarfism.

We elected to address this issue by creating mice with targeted mutations in their cytoplasmic domains which would eliminate key signaling pathways, then to observe 

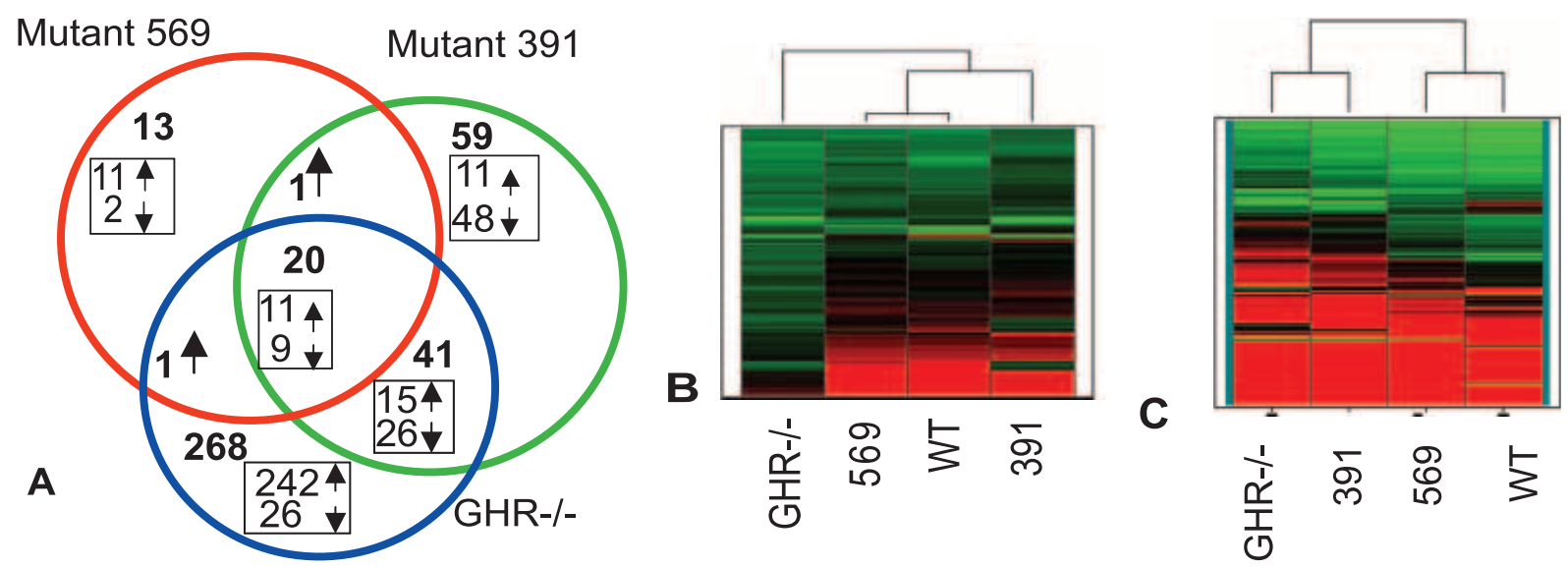

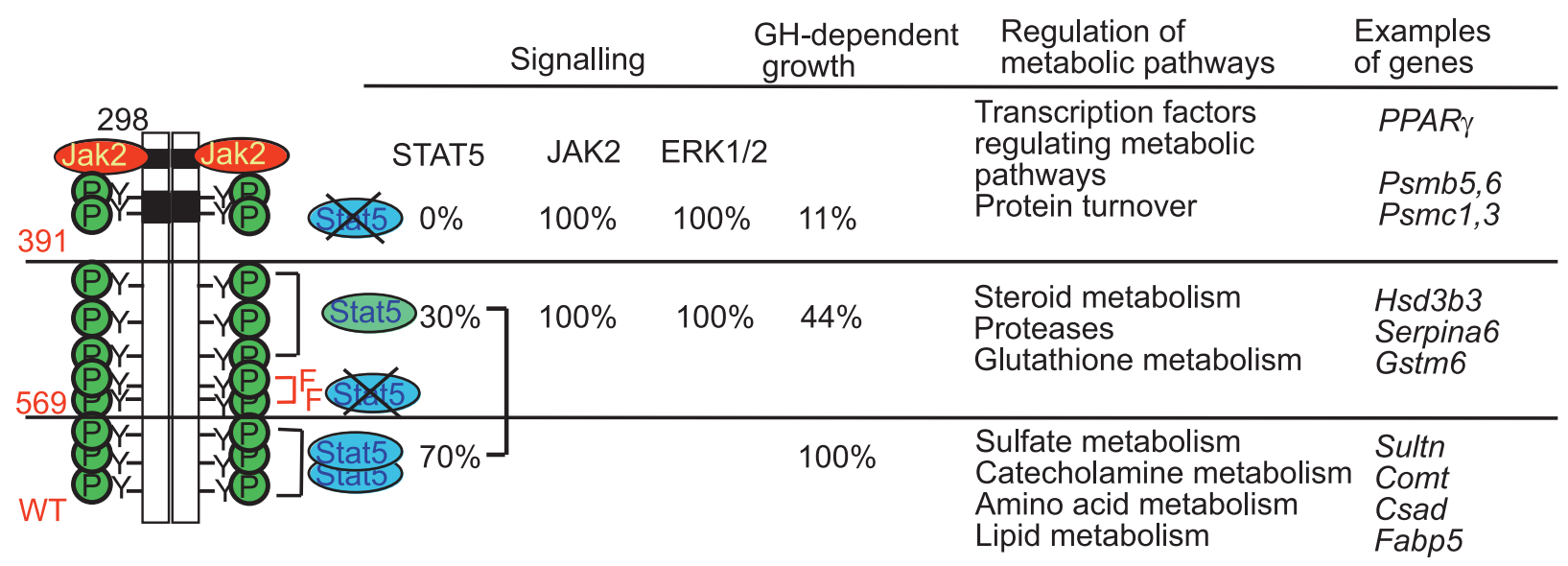

Figure 4 Global analysis of the GHR mutant mice using microarrays. (A) Venn diagram showing the number of genes differentially expressed in three mutant mice lines in comparison to the wild-type (WT) mice, with the number of genes up- and down-regulated in each group. Genes expressed in common between the mutants are showed by overlaps of the circles. (B, C) Hierarchical clustering shows that mutant 569 is most similar in its pattern of gene expression to the wild type and mutant 391 is similar to these two groups and $\mathrm{GHR}^{-/-}$mice cluster separately. Changes in signaling and $\mathrm{GH}$-dependent growth in GHR mutant mice are summarized in the lower panel. ERK, extracellular-signal-regulated kinase; PPAR, peroxisome-proliferator-activated receptor. From Rowland et al. (2005).

the effect of this on growth (Rowland et al. 2005). Currently, three such mice have been created involving (1) truncation at residue 569 with the two distal tyrosines converted to phenylalanine, to remove the majority of STAT5 signaling and the SHP2-binding site, originating from the distal 80 residues of the intact receptor, (2) truncation at 391, removing all STAT5 generation and presumably other signals in the central segment of the cytoplasmic domain and (3) mutation of the box 1 sequence, to abrogate JAK2 activation. This should leave the Src family kinase intact, and indicate the extent of GH action through JAK2.

The phenotypic consequences of these mutations can be seen in Fig. 3. It is clear that GHR signaling is essential for postnatal allometric growth, and that loss of
STAT5 signaling correlates progressively with loss of postnatal growth enhancement. The latter is in agreement with the recent study of Woelfe et al. (2003a), which has identified a functional STAT5 element in the second intron of the insulin-like growth factor (IGF)-I promoter, and a study by this group showing blockade of GH-dependent hepatic IGF-I generation in vivo with dominant negative STAT5 (Woelfe et al. 2003b). Further, specific STAT5b gene mutation results in major retardation of postnatal growth in humans (Kofoed et al. 2004). In our mice harboring GHR truncated at 569, loss of around $70 \%$ of STAT5 signaling results in around $50 \%$ loss of postnatal growth enhancement by $\mathrm{GH}$, and around $75 \%$ loss of circulating IGF-I. Interestingly, although the loss of 
Table 1 Simplified gene ontology classification of differentially expressed genes in the three transgenic lines in comparison to wild type

\begin{tabular}{|c|c|c|c|}
\hline & Mutant 569 & Mutant 391 & $\mathrm{GHR}^{-1-}$ \\
\hline Metabolism & 12 & 26 & 60 \\
\hline Cytochrome/Electron transport & 5 & 12 & 22 \\
\hline Glutathione/Antioxidant & 2 & 8 & 10 \\
\hline Transporters & 1 & 16 & 20 \\
\hline Proliferation/Differentiation/Growth & 2 & 10 & 30 \\
\hline Cell-to-cell interactions & 0 & 1 & 5 \\
\hline Transcription/Translation & 0 & 0 & 23 \\
\hline Signaling & 1 & 13 & 28 \\
\hline Protein processing & 4 & 18 & 17 \\
\hline Chaperones & 1 & 1 & 7 \\
\hline Ribosomal proteins & 0 & 1 & 17 \\
\hline Unclassified & 6 & 15 & 91 \\
\hline Total & 35 & 121 & 330 \\
\hline
\end{tabular}

hepatic IGF-I transcript is only around $30 \%$, because this is accompanied by major falls in IGF-binding protein 3 and acid labile unit, the clearance of circulating IGF-I increases, preferentially decreasing serum IGF-I. Truncation at residue 391 removes all STAT5a/b signaling, and results in a growth phenotype similar to, but significantly larger than, the complete GHR knockout (Zhou et al. 1997). The residual 15\% of GH-dependent growth evidently represents the STAT5a/b-independent component (Rowland et al. 2005).

We have used Affymetrix microarrays to define the transcripts regulated by the mutated receptor cytoplasmic domains, and compared these with the complete receptor deletion (Zhou et al. 1997), summarized in Fig 4. It is evident that the majority of the nearly 400 hepatic transcripts shown to be regulated by the GHR have their expression level controlled by the domain proximal to residue 391; that is, the core JAK2 signaling sequence. Further, many important regulatory genes are repressed by this proximal sequence. Removal of $70 \%$ of $\mathrm{STAT} 5 \mathrm{a} / \mathrm{b}$ generation has relatively little effect on the transcript profile, although complete removal of STAT5a/b generation (along with hepatic nuclear factor- $3 \beta$, and probably other pathways) has a substantially greater effect on transcript expression. Table 1 provides an overview of the functional classes of transcripts regulated by the different signaling domains. It is evident that, in keeping with the concept that the liver is the main target of $\mathrm{GH}$ action, a large number of hepatic functions are GH-regulated. Some of these, particularly in endothelial, hematopoietic and Kuppfer cells, may be altered as a result of altered IGF-I status rather than GH itself, but are nonetheless GHR-determined.

\section{Conclusion}

The more than 400 actions of GH are mediated by an array of signals triggered by the activated GHR. These include control of postnatal growth, hepatic metabolism, fertility and immune function, among others. The initiating event is relative rotation of receptor subunits within a constitutive receptor dimer. This aligns bound JAK2 and Src kinases, and their activation by transphosphorylation results in a cascade of signaling events. Postnatal growth is largely controlled by IGF-I, generated by STAT5a/b activation, although other pathways may be involved. A host of other hepatic transcripts are GH regulated, and these regulate many aspects of metabolic function.

\section{Acknowledgements}

Supported by an NHMRG (Australia) grant to M J W. The authors declare that there is no conflict of interest that would prejudice the impartiality of this scientific work.

\section{References}

Bernat B, Pal G, Sun M \& Kossiakoff AA 2003 Determination of the energetics governing the regulatory step in growth hormone-induced receptor homodimerization. PNAS $\mathbf{1 0 0}$ 952-957.

Brown RJ, Adams JJ, Pelekanos RA, Wan Y, McKinstry WJ, Palethorpe K, Seeber RM, Monks TA, Eidne KA, Parker MW \& Waters MJ 2005 A model for GH receptor activation based on subunit rotation within a receptor dimer. Nature Structural $\mathbb{F}^{\circ}$ Molecular Biology 12 814-821.

Chen C-M, Brinkworth R \& Waters MJ 1997 The role of receptor dimerization domain residues in $\mathrm{GH}$ signalling. Fournal of Biological Chemistry 272 5133-5140.

deVos AM, Ultsch M \& Kossiakoff AA 1992 Human growth hormone and extracellular domain of its receptor: crystal structure of the complex. Science 255 306-312.

Fuh G, Cunningham BC, Fukunaga S, Nagata S, Goeddel DV \& Wells JA 1992 Rational design of potent antagonists to the human growth hormone receptor. Science 256 1677-1680. 
Gent J, van Kerkhof P, Roza M, Bu G \& Strous GJ 2002 Ligand-independent growth hormone receptor dimerization occurs in the endoplasmic reticulum and is required for ubiquitin system-dependent endocytosis. PNAS 99 9858-9863.

Harding PA, Wang X, Okada S, Chen WY, Wan W \& Kopchick JJ 1996 Growth hormone $(\mathrm{GH})$ and a $\mathrm{GH}$ antagonist promote $\mathrm{GH}$ receptor dimerization and internalization. Fournal of Biological Chemistry 271 6708-6712.

Jiang J, Wang X, He K, Li X, Chen C-M, Sayeski PP, Waters MJ \& Frank SJ 2004 A conformationally sensitive GHR antibody; impact on GH signalling and proteolysis. Molecular Endocrinology 18 2981-2996.

Kofoed EM, Hwa V, Little B, Woods KA, Buckway CK, Tsubaki J, Pratt KL, Bezrodnik L, Jasper H, Tepper A et al. 2003 Growth hormone insensitivity associated with a STAT5b mutation. New England Fournal of Medicine 349 1139-1147.

Kubatzky KF, Ruan W, Gurezka R, Cohen J, Ketteler R, Watowich SS, Neumann D, Langosch D \& Klingmuller U 2001 Self assembly of the transmembrane domain promotes signal transduction through the erythropoietin receptor. Current Biology 11 110-115.

Pearce KH Jr, Cunningham BC, Fuh G, Teeri T \& Wells JA $1999 \mathrm{GH}$ binding affinity for its receptor surpasses the requirements for cellular activity. Biochemistry 38 81-89

Rowland JE, Lichanska AM, Kerr LM, White M, D'Aniello EM, Maher SL, Brown RJ, Teasdale R, Noakes PG \& Waters MJ 2005 In vivo analysis of growth hormone receptor signalling domains and their associated transcripts. Molecular Cell Biology 25 66-77.

Rowlinson SW, Behncken SN, Rowland JE, Clarkson RW, Strasburger CJ, Wu Z, Baumbach W \& Waters MJ 1998 Activation of chimeric and full-length growth hormone receptors by growth hormone receptor monoclonal antibodies. A specific conformational change may be required for full-length receptor signaling. Fournal of Biological Chemistry 273 5307-5314.
Schneider H, Chaovapong W, Matthews DJ, Karkaria C, Cass RT, Zhan H, Boyle M, Lorenzini T, Elliott SG \& Giebel LB 1997 Homodimerization of erythropoietin receptor by a bivalent monoclonal antibody triggers cell proliferation and differentiation of erythroid precursors. Blood $\mathbf{8 9} 473-482$.

Seubert N, Royer Y, Staerk J, Kubatzky KF, Moucadel V, Krishnakumar S, Smith SO \& Constantinescu SN 2003 Active and inactive orientations of the transmembrane and cytosolic domains of the erythropoietin receptor dimer. Molecular Cell 12 1239-1250.

van Kerkhof P, Smeets M \& Strous GJ 2002 The ubiquitinproteasome pathway regulates the availability of the $\mathrm{GH}$ receptor. Endocrinology 143 1243-1252.

Wan Y, Zheng YZ, Harris JM, Brown R \& Waters MJ 2003 Epitope map for a $\mathrm{GH}$ receptor agonist monoclonal antibody, MAb 263. Molecular Endocrinology 17 2240-2250.

Wan Y, McDevitt A, Shen B, Smythe ML \& Waters MJ 2004 Increased Site 1 affinity improves biopotency of porcine growth hormone: evidence against diffusion dependent receptor dimerization. Fournal of Biological Chemistry 279 44775-44784.

Woelfe J, Chia DJ \& Rotwein P 2003a Mechanisms of growth hormone $(\mathrm{GH})$ action. Identification of conserved Stat5 binding sites that mediate $\mathrm{GH}$-induced insulin-like growth factor-I gene activation. Fournal of Biological Chemistry 278 51261-51266.

Woelfe J, Billiard J \& Rotwein P 2003b Acute control of IGF-I gene transcription by growth hormone through Stat5b. Fournal of Biological Chemistry 278 22696-22702.

Zhou Y, Xu BC, Maheshwari HG, He L, Reed M, Lozykowski M, Okada S, Cataldo L, Coschigamo K, Wagner TE et al. 1997 A mammalian model for Laron syndrome produced by targeted disruption of the mouse growth hormone receptor/binding protein gene (the Laron mouse). PNAS 94 13215-13220.

Received 11 August 2005

Accepted 23 November 2005 